\title{
Patterns and outcomes of stroke thrombolysis in a large tertiary care hospital in Riyadh, Saudi Arabia
}

Ismail A. Khatri, MD, FAAN, Mohammed AlSkaini, SBN, AbdulRahman AlDayel, MBBS, Qamra AlBoqami, MPH, Emad Masuadi, PhD, Mufadhi AlShammari, SBN, Athal AlKhalaf, MD, PhD, Deema AlRasheed, SBN, Ali AlKhathaami, MBBS, MPH, FRCPC, Nasir AlOtaibi, MBBS, FRCPC, SSC-IM, Maisoun Tarawneh, RN, Khloud AlHizan, RN.

\begin{abstract}

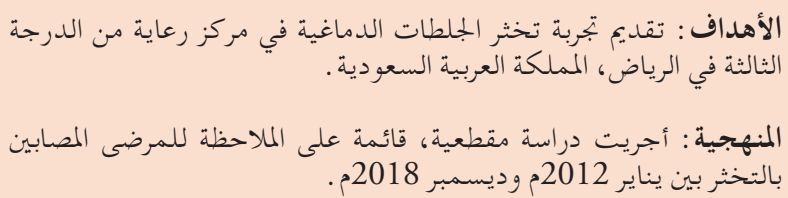

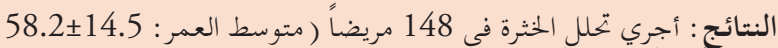

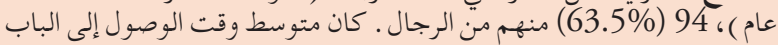

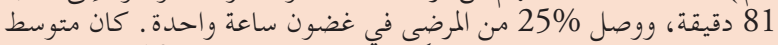

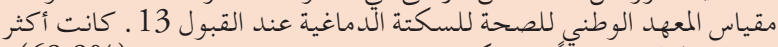

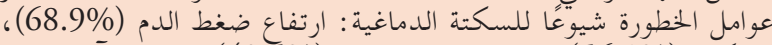

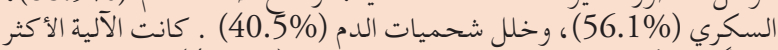

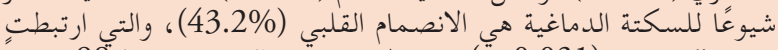

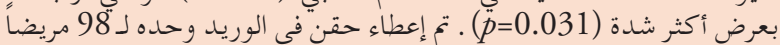

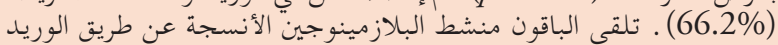

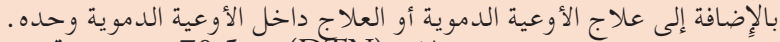

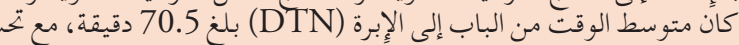

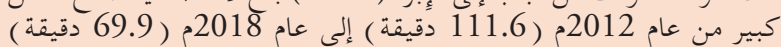
(م)

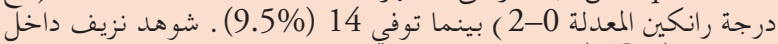

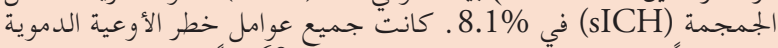

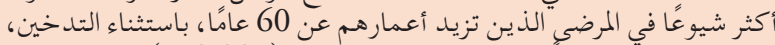

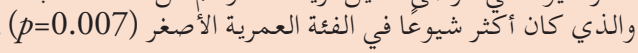

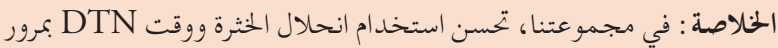

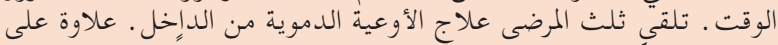

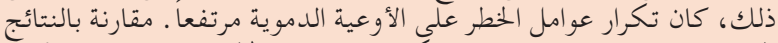

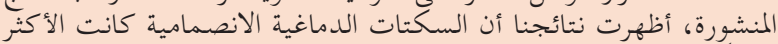

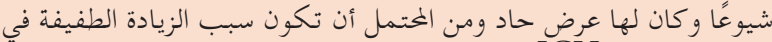

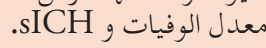

Objectives: To present the experience on stroke thrombolysis of a tertiary care center in Riyadh, KSA.

Methods: Cross-sectional, observational study of patients thrombolyzed between January 2012 and December 2018.

Results: Thrombolysis was performed in 148 patients (mean age: $58.2 \pm 14.5$ years), $94(63.5 \%)$ of them were men. The median onset-to-door time was 81 minutes, and $25 \%$ of the patients arrived within 1 hour. The median National Institute of Health Stroke
Scale score upon admission was 13 . Hypertension $(68.9 \%)$, diabetes $(56.1 \%)$, and dyslipidemia $(40.5 \%)$ were the most common risk factors for stroke. The most common mechanism of stroke was cardioembolism (43.2\%), which was associated with a more severe presentation $(p=0.031)$. Intravenous thrombolysis alone was given to 98 patients $(66.2 \%)$; the rest received intravenous tissue plasminogen activator plus endovascular therapy or endovascular therapy alone. The median door-to-needle (DTN) time was $70.5 \mathrm{~min}$, with a significant improvement from 2012 (111.6 minutes) to 2018 (69.9 minutes) $(p<0.001)$. Among the patients, $53(35.8 \%)$ showed a good outcome (with a modified Rankin score of $0-2$ ) whereas $14(9.5 \%)$ died. Symptomatic intracranial hemorrhage $(\mathrm{sICH})$ was seen in $8.1 \%$. All vascular risk factors were more common in patients aged $>60$ years, except smoking, which was more common in the younger age group $(p=0.007)$.

Conclusion: In our cohort, the utilization of thrombolysis and the DTN time improved over time. One-thirds of the patients received endovascular treatment. Moreover, the frequency of the vascular risk factors was high. Compared with the published findings, our results showed that cardioembolic strokes were the most frequent and had severe presentation and were likely the cause of the slight increase in mortality and sICH.

Neurosciences 2021; Vol. 26 (2): 199-206 doi: 10.17712/nsj.2021.2.20200171

From the Division of Neurology (Khatri, AlSkaini, AlDayel, AlShammari, AlKhalaf, AlRasheed, AlKhathaami, AlOtaibi), Department of Medicine, Department of Nursing (Tarawneh, AlHizan), King Abdulaziz Medical City, Ministry of National Guard Health Affairs, from the College of Medicine (Khatri, Masuadi, AlKhathaami, AlOtaibi), King Saud bin Abdulaziz University for Health Sciences, from King Abdullah International Medical Research Center (Khatri, Masuadi, AlKhathaami, AlOtaibi), from the College of Public Health (AlBoqami), King Saud bin Abdulaziz University for Health Sciences, Riyadh, Kingdom of Saudi Arabia

Received 24th November 2020. Accepted 23rd January 2021.

Address correspondence and reprint request to: Dr. Ismail A. Khatri, Division of Neurology, Department of Medicine, King Abdulaziz Medical City, Ministry of National Guard Health Affairs, Riyadh, Kingdom of Saudi Arabia.E-mail: ismailkhatri@yahoo.com ORCID ID: orcid.org/0000-0002-0218-1840 
A cute ischemic stroke remains a major cause of morbidity and mortality worldwide. Intravenous (IV) thrombolysis is the standard of care in eligible stroke patients since 1995 after the publication of the National Institute of Neurological Disorders and Stroke (NINDS) recombinant tissue plasminogen activator [alteplase] (rtPA) trial. ${ }^{1}$ In appropriately selected patients with acute ischemic stroke and large vessel occlusion (LVO), mechanical thrombectomy (MT) significantly improved the patients' functional independence without increasing the mortality rate or hemorrhagic complications. ${ }^{2}$ Despite the proven efficacy and relative safety of IV thrombolysis and MT, they remain underutilized. In our center, thrombolysis was utilized in $8.6 \%$ of all ischemic strokes between February 2016 and July 2018 and in 29\% of patients who arrived within the therapeutic window. ${ }^{3}$ Although acute stroke care has significantly improved in Saudi Arabia in the past decade, it remains far from being optimal. In a recent survey, nearly half of the Saudi emergency physicians considered the role of rtPA in stroke as controversial, whereas $37 \%$ presume that the efficacy of rtPA remains unproven. ${ }^{4} \mathrm{~A}$ recent survey showed that among the neurologists in Saudi Arabia, $21.3 \%$ had never used rtPA in acute stroke, $82.4 \%$ believed that the delayed presentation to a hospital was the most important barrier to the use of intravenous rtPA, and $50 \%$ considered that the unclear time of onset was the barrier to the use of thrombolysis. ${ }^{5}$ Another survey showed that among the neurology residents in Saudi Arabia, 79\% reported lack of good knowledge about IV thrombolysis and 53.1\% expressed lack of confidence in using IV thrombolysis. ${ }^{6}$ The use of IV thrombolysis and MT has gained momentum in Saudi Arabia, but the published literature on the patterns and outcomes of thrombolysis remains scant. Recently, results from the Safe Implementation of Treatment in Stroke (SITS)-Middle East and North African (MENA) registry for IV thrombolysis were published, ${ }^{7}$ and a report from Dubai described the use and outcomes of thrombolysis in 176 patients. $^{8}$

A stroke unit was formally established in our tertiary care center in 2012, although thrombolysis treatment was already offered prior to that. Here, we report our experience on thrombolysis in acute ischemic stroke from January 2012 to December 2018. We presume that this report is the first of its kind in Saudi Arabia.

Disclosure. Authors have no conflict of interests, and the work was not supported or funded by any drug company.
Methods. This work is a cross-sectional, retrospective chart review approved by the Institutional Review Board (IRB) of King Abdullah International Medical Research Center (KAIMRC). The patients who underwent thrombolysis at King Abdulaziz Medical City, Ministry of National Guard Health Affairs, Riyadh, KSA, between January 2012 and December 2018 were identified from hospital records. The requirement for informed consent was waived by the IRB. In this study, patients were selected in a non-random, consecutive sampling fashion. However, those patients who received thrombolysis without a proper stroke code activation and those who had stroke while in hospital (inpatient strokes) were excluded. In our center, stroke code is activated for patients who arrive with stroke symptoms of less than 6 hours duration. These were the patients who required thrombolysis outside of routine practice based on their clinician's discretion. Most of the excluded patients had basilar thrombosis and had endovascular intervention outside the routine time window. These patients were found to have acute vascular thrombosis during routine neurology consultations, hence the door-to-needle (DTN) time, door-to-groin (DTG) time and other intervention times were not consistent with routine stroke code activation.

The standard dose of $0.9 \mathrm{mg} / \mathrm{kg}$ of alteplase was used for intravenous thrombolysis. The dose of intraarterial alteplase, when used, was on the discretion of interventional radiologist, and was not to exceed 20 mg. The intravenous thrombolysis was performed up to 4.5 hours from symptoms onset, whereas most of the endovascular procedures were performed within 6 hours of symptoms onset.

Stroke severity was measured using National Institute of Health Stroke Scale (NIHSS) scores at arrival, 48 hours, with any sudden change in clinical status, and at the time of discharge. Outcome was measured with modified Rankin Scale (mRS) at the time of discharge.

Symptomatic intracerebral hemorrhage (sICH) was defined according to SITS-MOST definition which included parenchymal hemorrhage type 2 (PH2) with neurological deterioration indicated by increase in NIHSS score of 4 points or more from baseline or lowest value within past week or any hemorrhage leading to death. ${ }^{9}$ Cerebral edema was defined based on radiological images showing midline shift or cerebral herniation. All images and clinical assessment were reviewed by trained neurologist (stroke fellow) to ascertain the type of hemorrhage, evidence of cerebral edema and neurological deterioration.

Data on predefined variables was collected and analyzed using the Statistical Package for Social Sciences 
Table 1 - Demographic features, stroke type, risk factors, and time indicators for thrombolyzed patients.

\begin{tabular}{|c|c|}
\hline \multicolumn{2}{|l|}{ Patients $(n=148)$} \\
\hline Mean age $( \pm S D)$ in years & $58.2 \pm 14.5$; range $22-87$ years \\
\hline Male (\%) & $94(63.5)$ \\
\hline Median NIHSS score at admission (inter-quartile range) & $13(8-18)$ \\
\hline \multicolumn{2}{|l|}{ TOAST classification n (\%) } \\
\hline Cardioembolic & $64(43.2)$ \\
\hline Large artery & $18(12.2)$ \\
\hline Small vessel & $15(10.1)$ \\
\hline Other determined & $1(0.7)$ \\
\hline Undetermined & $41(27.7)$ \\
\hline \multicolumn{2}{|l|}{ Vascular risk factors $n(\%)$} \\
\hline Hypertension & $102(68.9)$ \\
\hline Diabetes mellitus & $83(56.1)$ \\
\hline Dyslipidemia & $60(40.5)$ \\
\hline Ischemic heart disease & $31(20.9)$ \\
\hline Prior stroke & $23(15.5)$ \\
\hline Congestive heart failure & $16(10.8)$ \\
\hline Smoking & $36(24.3)$ \\
\hline Atrial fibrillation & $40(27.0)$ \\
\hline \multicolumn{2}{|l|}{ Time indicators in minutes - Median (IQR) } \\
\hline Symptoms onset-to-door time & $81(54.5-115)$ \\
\hline Door-to-stroke code activation & $7(4-14)$ \\
\hline Stroke team arrival from stroke code activation & $4(2-8)$ \\
\hline Door-to-CT scan & $28(21-39)$ \\
\hline Door-to-needle time & $70.5(57-94)$ \\
\hline Door-to-groin time for endovascular therapy & $156(122-212)$ \\
\hline
\end{tabular}

(SPSS) software version 23.0. Descriptive statistics for categorical data are presented as frequencies and percentages. Numerical data are presented as means and standard deviations. Pearson chi-square test was used to analyze statistical significance. Statistical significance was set at a $p$-value of $<0.05$.

Results. A total of 3,070 patients were admitted to our stroke service, including hemorrhagic strokes and stroke mimics between January 2012 and December 2018; of these patients, 164 (5.3\% of all strokes, and $8.6 \%$ of all ischemic strokes) received thrombolysis.

Based on the inclusion and exclusion criteria, 148 patients were included in the final analysis. Among the 148 patients, $94(63.5 \%)$ were men. The mean age of the entire sample was $58.2( \pm 14.5)$ years, and no significant difference was observed between the mean ages of the males $(57.1 \pm 14.0)$ and the females $(60.2 \pm 15.3)(p=0.212)$. The median National Institute of Health Stroke Scale (NIHSS) score at presentation was 13 with an inter-quartile range (IQR) of 8-18.

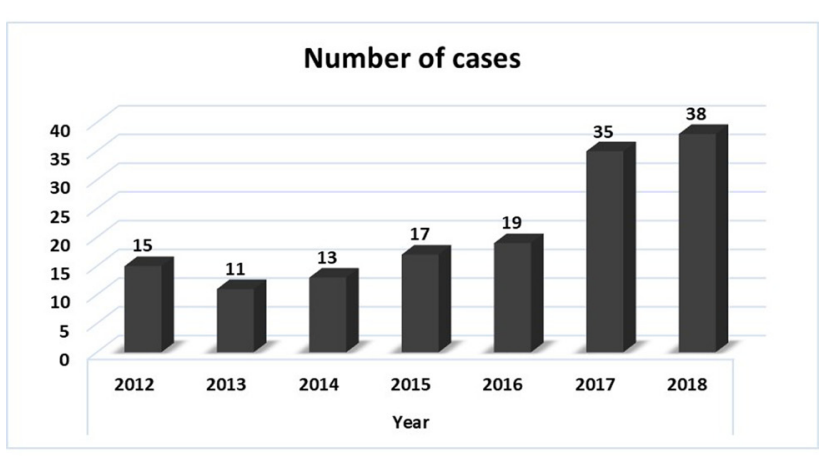

Figure 1 - Increasing utilization of thrombolysis over time.

The demographic features, vascular risk factors, TOAST classification of stroke, and time indicators are summarized in Table 1. Hypertension (68.9\%), diabetes mellitus $(56.1 \%)$, and dyslipidemia (40.5\%) were the commonest vascular risk factors. Cardioembolism was 


\section{Yearly trend for thrombolytic pattern}

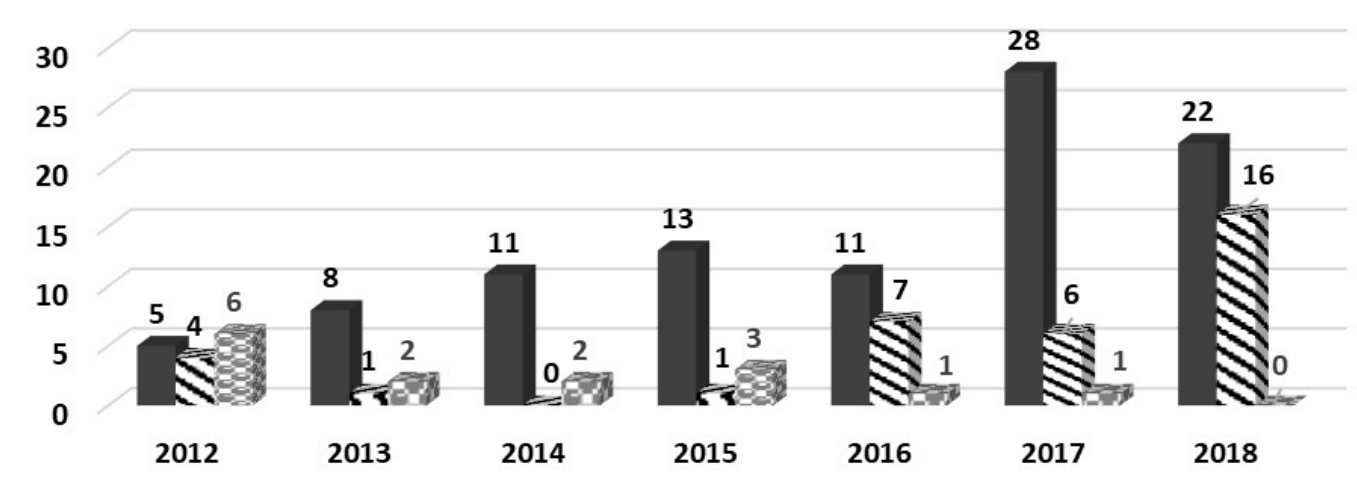

— Intravenous alone $\approx$ Intravenous plus intraarterial/endovascular ${ }_{i}$ Intraarterial/endovascular alone

Figure 2 - Yearly trend for thrombolytic pattern (intravenous alone, intravenous plus intraarterial/endovascular, or intraarterial/endovascular alone).

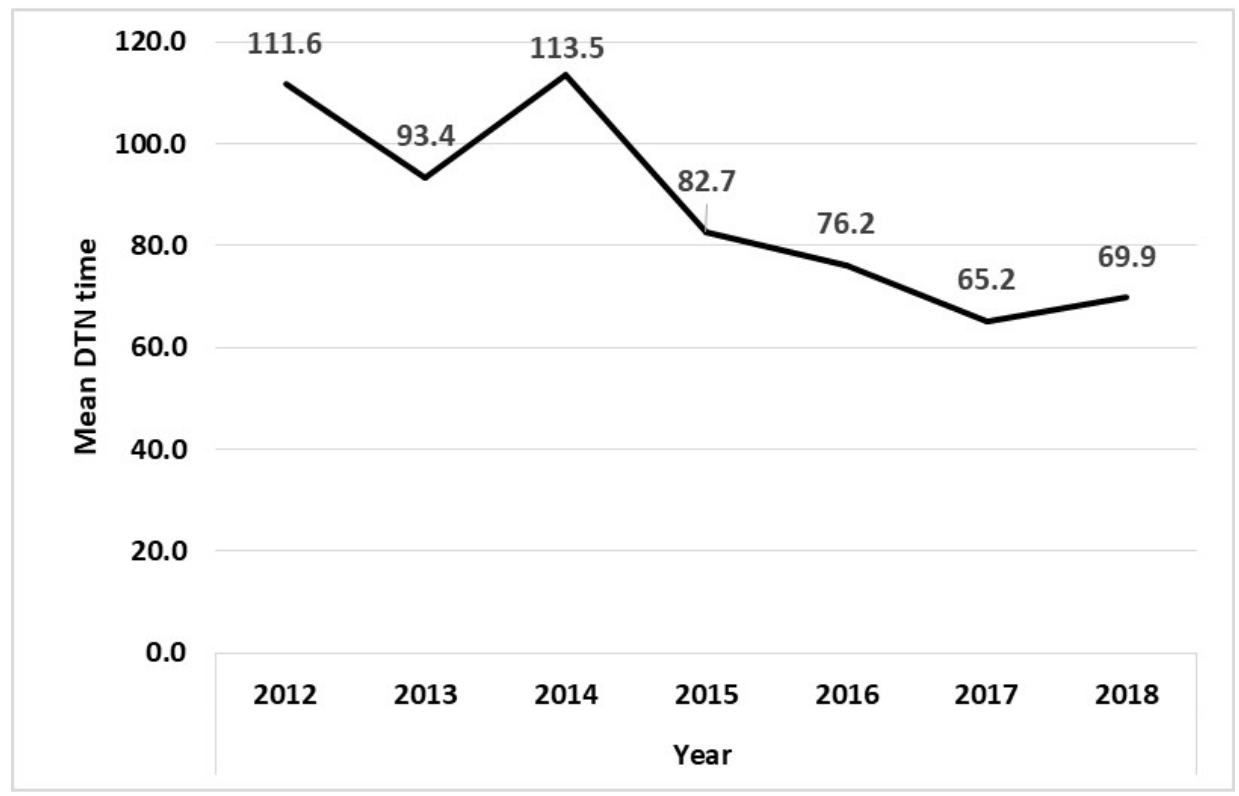

Figure 3 - Improving trend for door-to-needle (DTN) time.

the commonest mechanism of stroke (43.2\%) and was associated with more severe presentation $(49 \%$ versus $31 \%)$ with a $p$-value of 0.031 . Stroke of undetermined etiology was seen in $27.7 \%$ of the patients, whereas large artery stroke was seen in $12.2 \%$ of the patients.

The number of patients who received thrombolytic therapy increased gradually over time, with the highest year-to-year increase between 2016 and 2017 (Figure 1). Only 15 out of 486 (3.1\%) patients received thrombolysis in 2012, compared with the 35 out of $470(7.4 \%)$ patients in 2017 and the 38 out of 494 (7.7\%) patients in 2018. IV thrombolysis alone was used in 98 (66.2\%) patients, whereas the rest received 
Table 2 - Efficacy and discharge outcomes of thrombolyzed patients.

\begin{tabular}{lcc}
\hline Outcomes & n (\%) & $P$-value \\
\hline$m R S$ at discharge $n(\%)$ & & \\
$0-2$ & $53(35.8)$ & \\
$3-5$ & $52(35.1)$ & \\
6 & $14(9.5)$ & \\
Missing & $29(19.6)$ & \\
NIHSS at admission $(\mathrm{n}=141)$ & $13(8-18)$ & $<0.001$ \\
Median (IQR) & & \\
NIHSS at discharge $(\mathrm{n}=84)$ & $3(1-9)$ & \\
Median (IQR) & & \\
sICH n (\%) & $12(8.1)$ & \\
In-hospital death n $(\%)$ & $14(9.5)$ & \\
\hline
\end{tabular}

either a combination of IV thrombolysis and MT or MT alone. The use of endovascular treatment increased significantly from 2016 to 2018 (Figure 2).

The time indicators for thrombolysis are shown in Table 1. The median symptom onset-to-door time was 81 minutes. A quarter (25\%) of the patients arrived within 1 hour from the symptom onset. The median door-to-CT scan time was 28 minutes and the median door-to-needle (DTN) time was 70.5 minutes. The mean DTN time improved over time, that is, from 111.6 minutes in 2012 to 65.2 minutes in 2017 and 69.9 minutes in 2018 (Figure 3). For the patients who underwent endovascular treatment, the median doorto-groin time was 156 minutes (IQR 122-212).

The discharge outcomes are summarized in Table 2. Some of the outcome parameters, including discharge modified Rankin scale (mRS) and discharge NIHSS score, were missing in some patients. A total of 53 $(35.8 \%)$ patients had an mRS of 0-2 at discharge, whereas $52(35.1 \%)$ patients had an mRS of 3-5 at discharge. There were $14(9.5 \%)$ in-hospital deaths among the thrombolyzed patients. Unfortunately, the discharge mRS of $29(19.6 \%)$ patients was not recorded. Symptomatic intracranial hemorrhage $(\mathrm{sICH})$ was seen in $12(8.1 \%)$ patients. Stroke onset to time of presentation, DTN time, NIHSS score at presentation, age, gender, and vascular risk factors did not affect the occurrence of sICH. The median discharge NIHSS score was 3 (IQR 1-9), whereas the admission NIHSS score was 13 (IQR 8-18) $(p \leq 0.001)$.

All vascular risk factors were more common in patients aged 60 years and above, except for smoking, which was more common in patients younger than 60 years, as shown in Table 3. Patients older than 60 years presented with more severe strokes (with an
NIHSS score of $>15$ ) compared with younger patients $(p=0.018)$. The discharge mRS and discharge NIHSS scores did not differ with age.

Females were more likely to be admitted to ICU $(96 \%$ vs. $75 \%) \quad(p=0.001)$ and were more likely to experience asymptomatic hemorrhage (24\% vs. $10 \%)$ $(p=0.017)$ than males. The vascular risk factors in stroke did not differ between males and females. Moreover, gender did not exert any significant effect on stroke severity at presentation $(p=0.236)$ or on discharge $\mathrm{mRS}$ $(p=0.575)$. No difference in the time of arrival to ER $(p=0.26)$ nor in the DTN time $(p=0.119)$ was observed between males and females.

Discussion. Thrombolysis has remained underutilized in the management of acute ischemic stroke despite the availability of efficacy data gathered for more than 2 decades. The Get with the Guideline (GWTG)-Stroke initiative of the American Heart Association resulted in the improvement in the use of IV rtPA from $4 \%$ to $7 \%$ from 2003 to $2011 .{ }^{10}$ In our cohort, the IV rtPA utilization increased to more than twice from $3.1 \%$ in 2012 to $7.7 \%$ in 2018 in all stroke admissions. Although this improvement is encouraging, the optimal utilization of IV thrombolysis remains challenging. A retrospective study involving more than 60,000 patients in a national registry in the USA showed that patients of older age, with milder strokes, of the female gender, and belonging to minority groups were likely to be undertreated. ${ }^{11}$ The DTN time improved from more than 110 minutes in 2012 to less than 70 minutes in 2018. We implemented several changes in our stroke code pathway as quality improvement project from 2013 onwards. These changes targeted various timelines that resulted in the significant improvement in the DTN time. The GWTG-Stroke initiative also reported improvement in the DTN time from 82 minutes to 71 minutes when guidelines were implemented. ${ }^{10}$ The mean DTN times reported in the study from Dubai was 64.1 minute $^{8}$ whereas that reported in the SITS-MENA registry was 54 minutes. $^{7}$

Endovascular thrombectomy hasbecomethestandard of care for patients with LVO-related strokes. ${ }^{12}$ Multiple randomized controlled trials published in 2015 had established the efficacy of endovascular/mechanical thrombectomy in LVO with no significant increase in sICH. ${ }^{2,13}$ Before the publication of these trials, we were already using intra-arterial thrombolysis/endovascular thrombectomy in select patients in our center. After the recent trials were published, the number of patients undergoing endovascular thrombectomy increased significantly. In our cohort, the door-to-groin time 
was less than the optimal. We could not perform a subgroup analysis due to the small number of patients who underwent endovascular thrombectomy alone.

Most of our patients had a moderately severe stroke with a median NIHSS score of 13. In the SITS-MENA registry, the median NIHSS score of the patients who received thrombolysis was $12,{ }^{7}$ whereas that for the observational cohort was $8 .{ }^{14}$ The mean NIHSS score of the patients who received thrombolysis in the Dubai study was 12 . These numbers are consistent with that reported in the GWTG-Stroke registry, wherein the median NIHSS score of patients who received IV thrombolysis was $12 .{ }^{10}$ In our cohort, more than one-third of the patients suffered from cardioembolic stroke, which may have contributed to the higher NIHSS score at presentation. As suggested in the GWTG-Stroke registry, the patients with milder strokes may have been undertreated; ${ }^{11}$ hence, the studies that included only those patients who received IV thrombolysis had reported higher median NIHSS scores.

Nearly two-thirds of the patients who received thrombolysis at our center were male. This gender difference was much higher in the study from Dubai wherein $82.8 \%$ of the thrombolyzed patients were male. ${ }^{8}$ In the SITS-MENA registry, $72 \%$ of the patients were male, whereas $53.6 \%$ of the patients were male in the non-MENA region. ${ }^{7}$ The higher rate of thrombolysis in males is not a regional phenomenon. The GWTG-Stroke registry showed that the female gender was an independent determinant of under-treatment with thrombolysis. ${ }^{11}$

Stroke occurs at a younger age in Saudi Arabia and Middle East. The mean age of our patients was 58.2 years. A recent study conducted in a university hospital in Saudi Arabia reported that the mean age of the investigated stroke patients was 60.4 years. ${ }^{15}$ The median age of the thrombolyzed patients included in the in SITS-MENA registry was 55 years, whereas the median age reported in the non-MENA region was 73 years. ${ }^{7}$ In the Dubai cohort, the mean age was 50.8 years, which is lower than that in our cohort. ${ }^{8}$ In a review of 30 studies conducted in the Middle East and involving more than 21,000 patients, a median age of less than 65 years was reported in two-thirds of the studies. ${ }^{16}$ A study in Saudi Arabia reported a stroke incidence of 108.05 strokes per 100,000 population aged 50-59 years compared with the overall incidence of 57.64 strokes per 100,000 population in the Aseer region in Saudi Arabia. ${ }^{17}$

There is an staggering burden of stroke risk factors in the Saudi and Middle Eastern populations. A university hospital-based study in Saudi Arabia reported that among the stroke patients, $61.4 \%$ had hypertension, $50.8 \%$ had diabetes mellitus, and $20.6 \%$ had atrial fibrillation. ${ }^{15}$ These numbers have dramatically increased in the past two decades. The data on 1000 stroke patients reported in the Saudi Stroke Data Bank showed that among those patients, $52 \%$ had hypertension, $42 \%$ had diabetes, $10 \%$ had atrial fibrillation, and $10 \%$ were smokers. ${ }^{18}$ Moreover, a review of 30 studies involving stroke patients from various regions of the Middle East reported that among the investigated patients, $62.1 \%$ had hypertension, 33.1\% had diabetes mellitus, 36.8\% had dyslipidemia, and $13.6 \%$ had atrial fibrillation. ${ }^{16}$ The prevalence of these classical risk factors in our cohort were similar to that in the abovementioned reports or even higher. In our patients, more than 50\% had diabetes and $27 \%$ had atrial fibrillation. All of these risk factors were more prevalent in patients 60 years old and above, except smoking, which was more prevalent in the younger age group.

Approximately $20 \%-30 \%$ of all stroke cases can be attributed to cardioembolism and are usually more disabling. ${ }^{19}$ Cardioembolic stroke was the commonest stroke mechanism in our cohort, and it may have been promoted by the increased frequency of atrial fibrillation, which was observed in more than one quarter of the patients. Cardioembolism was not only the commonest mechanism, but was also associated with more severe strokes. Another possible explanation for cardioembolic strokes being a common condition in our cohort is that patients with less severe stroke who likely had small vessel disease were less often thrombolyzed. However, we could not confirm this correlation from the available data. Cardioembolic stroke was also common in the Dubai cohort; however, small vessel disease was the commonest stroke mechanism in their series. ${ }^{8}$ In the SITS-MENA observational registry, large vessel disease was considered the commonest mechanism in $46 \%$ of all ischemic stroke cases. ${ }^{14}$ Cardioembolic strokes were seen in approximately $20 \%$ of patients in the eastern region of Saudi Arabia. ${ }^{15}$

More than one-thirds of our patients were functionally independent at the time of discharge, with another one-thirds partly or fully dependent. In the Dubai cohort, more than two-thirds of the patients were discharged with an mRS of 2 or lower. ${ }^{8}$ In comparison, the SITS-MENA registry reported an mRS of 0-2 in $53 \%$ of the patients. ${ }^{7}$ Outcome data was missing in $19.6 \%$ of our patients, and the availability of which could have possibly improved the overall functional independence outcome. 
The mortality in our cohort was $9.5 \%$, which is higher than the $6.8 \%$ mortality reported in the Dubai cohort ${ }^{8}$ and the $2.3 \%$ mortality reported in the SITS-MENA registry. ${ }^{7}$ A considerably higher mortality of $17 \%$ at three months was reported in the original NINDS rtPA trial [NINDS rtPA]. In the recent studies from Saudi Arabia, the overall in-hospital stroke mortality rates were $9.7 \%{ }^{20}$ and $11.1 \% .{ }^{21}$ Moreover, sICH was seen in $8.1 \%$ of our patients, a rate that is higher than that reported in the NINDS rtPA trial (6.4\%), in the SITS-MENA registry $(<1.4 \%)$, and in the Dubai cohort $(6.9 \%) ., 7-8$ The increased sICH frequency and mortality rate may be related to the increased frequency of cardioembolic strokes, which may lead to hemorrhagic conversion as well as to a poor outcome. ${ }^{19,22}$ Compared with embolic stroke of uncertain source, cardioembolic strokes are not only associated with poor outcome but also with higher recurrence. ${ }^{22}$

We believe that this is the first report on thrombolysis utilization patterns and outcomes from Saudi Arabia. With a dedicated stroke unit, many of the thrombolysis targets were achieved, although all of the outcomes were not comparable to those achieved by the developed nations. Our study emphasizes the need for continuous improvement in the processes to achieve better outcomes. This study should serve as a precedent for other centers to share their experiences on thrombolysis and should eventually lead to the establishment of a national registry.

Our study has several limitations. Given that this work is a single-center study, the results cannot be generalized to the entire country. Data on the outcome was missing in several patients and could not be determined due to the retrospective nature of the study. Another limitation of the retrospective data collection is that we could not ascertain whether there were any protocol violations in the administration of $\mathrm{rtPA}$ particularly in patients who had unfavorable outcomes. Moreover, a long-term follow up on these patients was not conducted.

Conclusions and recommendations. In our cohort of thrombolyzed stroke patients, the utilization of thrombolysis has improved over time. Similarly, the DTN time improved over the years. The frequency of vascular risk factors was considerably high in our cohort, warranting urgent attention. The mortality rate and sICH frequency slightly increased possibly due to the increased frequency of cardioembolic strokes.

We expect that our study will prompt other centers to report their experiences on thrombolysis. A national registry or database for stroke thrombolysis is a current need. Future multicenter, multicity studies on stroke will considerably improve our understanding of stroke care in Saudi Arabia and will also help set optimal targets and goals in this field.

Acknowledgements. The authors gratefully would like to thank Scribendi Inc. Chatham, Ontario N7M ON3, Canada for English language editing.

\section{References}

1. National Institute of Neurological Disorders and Stroke rt-PA Stroke Study Group. Tissue plasminogen activator for acute ischemic stroke. N Engl J Med 1995; 333: 1581-1587.

2. Lambrinos A, Schaink AK, Dhalla I, Krings T, Casaubon LK, Sikich N, et al. Mechanical thrombectomy in acute ischemic stroke: a systematic review. Can J Neurol Sci 2016; 43: 455-60.

3. Al Khathaami AM, Al Bdah B, Tarawneh M, Alskaini M, Alotaibi F, Alshalan A, et al. Utilization of intravenous tissue plasminogen activator and reasons for nonuse in acute ischemic stroke in Saudi Arabia. J Stroke Cerebrovasc Dis 2020; 29: 104761.

4. Al Khathaami AM, Aloraini $H$, Almudlej S, Al Issa H, Elshammaa N, Alsolamy S. Knowledge and attitudes of Saudi emergency physicians toward t-PA use in stroke. Neurol Res Int 2018; 2018: 3050278.

5. Alanazy MH, Barakeh RB, Asiri A, Edrees MF, Abuzinadah AR, Aljafen BN, et al. Practice patterns and barriers for intravenous thrombolysis: a survey of neurologists in Saudi Arabia. Neurol Res Int 2018; 2018: 1695014.

6. Alharbi FA, Maghfuri NM, Abdu DM, Redine MY. Saudi neurology residents' knowledge and attitudes toward intravenous thrombolysis in patients with acute ischemic stroke. J Family Med Prim Care 2020; 9: 192-196.

7. Al-Rukn S, Mazya M, Akhtar N, Hashim H, Mansouri B, Faouzi B, et al. Stroke in the Middle-East and North Africa: a 2-year prospective observational study of intravenous thrombolysis treatment in the region. Results from the SITS-MENA Registry. Int J Stroke 2019; 8: 1747493019874729.

8. Khan M, Hashim H, Nisa Z, Kamran SH, Alrukn S. Thrombolysis for acute ischemic stroke: experience in Dubai, and comparison of Arab with non-Arab population. J Neurol Stroke 2016; 4: 00156.

9. Wahlgren N, Ahmed N, Dávalos A, Ford GA, Grond M, Hacke W, et al; SITS-MOST investigators. Thrombolysis with alteplase for acute ischaemic stroke in the Safe Implementation of Thrombolysis in Stroke-Monitoring Study (SITS-MOST): an observational study. Lancet 2007; 369: 275-282.

10. Schwamm LH, Ali SF, Reeves MJ, Smith EE, Saver JL, Messe S, et al. Temporal trends in patient characteristics and treatment with intravenous thrombolysis among acute ischemic stroke patients at Get with the Guidelines-Stroke hospitals. Circ Cardiovasc Qual Outcomes 2013; 6: 543-549.

11. Messé SR, Khatri P, Reeves MJ, Smith EE, Saver JL, Bhatt $\mathrm{DL}$, et al. Why are acute ischemic stroke patients not receiving IV tPA? Results from a national registry. Neurology 2016; 87: 1565-1574.

12. Turc G, Bhogal P, Fischer U, Khatri P, Lobotesis K, Mazighi M, et al. European Stroke Organisation (ESO) - European Society for Minimally Invasive Neurological Therapy (ESMINT) Guidelines on mechanical thrombectomy in acute ischaemic stroke endorsed by Stroke Alliance for Europe (SAFE). Eur Stroke J 2019; 4: 6-12. 
13. Goyal M, Menon BK, van Zwam WH, Dippel DW, Mitchell PJ, Demchuk AM, et al; HERMES collaborators. Endovascular thrombectomy after large-vessel ischaemic stroke: a metaanalysis of individual patient data from five randomised trials. Lancet 2016; 387: 1723-1731.

14. Rukn SA, Mazya MV, Hentati F, Sassi SB, Nabli F, Said Z, et al. Stroke in the Middle-East and North Africa: a 2-year prospective observational study of stroke characteristics in the region-results from the Safe Implementation of Treatments in Stroke (SITS)-Middle-East and North African (MENA). Int J Stroke 2019; 14: 715-722.

15. Zafar A. A review of 500 patients with acute stroke admitted to the University Hospital of Saudi Arabia. Similarities and differences compared with the published literature. Funct Neurol 2018; 33: 73-79.

16. Behrouz R, Powers CJ. Epidemiology of classical risk factors in stroke patients in the Middle East. Eur J Neurol 2016; 23: 262-269.

17. Alhazzani AA, Mahfouz AA, Abolyazid AY, Awadalla NJ, Aftab R, Faraheen A, et al. Study of stroke incidence in the Aseer Region, Southwestern Saudi Arabia. Int J Environ Res Public Health 2018; 15: 215.
18. AlRajeh S, Awada A. Stroke in Saudi Arabia. Cerebrovasc Dis 2002; 13: 3-8.

19. O'Carroll CB, Barrett KM. Cardioembolic stroke. Continuum (Minneap Minn) 2017; 23: 111-132.

20. Alhazzani AA, Mahfouz AA, Abolyazid AY, Awadalla NJ, Katramiz K, Faraheen A, et al. In hospital stroke mortality: rates and determinants in Southwestern Saudi Arabia. Int J Environ Res Public Health 2018; 15: 927.

21. Almekhlafi MA. Trends in one-year mortality for stroke in a tertiary academic center in Saudi Arabia: a 5-year retrospective analysis. Ann Saudi Med 2016; 36: 197-202.

22. Arauz A, Morelos E, Colín J, Roldán J, Barboza MA. Comparison of functional outcome and stroke recurrence in patients with embolic stroke of undetermined source (ESUS) vs. cardioembolic stroke patients. PLoS One 2016; 11: e0166091.

\section{Supplements}

* Supplements will be considered for work including proceedings of conferences or subject matter covering an important topic

* Material can be in the form of original work or abstracts.

* Material in supplements will be for the purpose of teaching rather than research.

* The Guest Editor will ensure that the financial cost of production of the supplement is covered.

* Supplements will be distributed with the regular issue of the journal but further copies can be ordered upon request.

* Material will be made available on Saudi Medical Journal website 